\title{
EVALUATION AND CORRELATION OF CARDIOVASCULAR DYSFUNCTION IN NONALCOHOLIC CIRRHOTIC PATIENTS WITH ITS SEVERITY
}

\author{
Subhas Bhuin'1, Rina Mohanty²
}

1 Junior Resident, Department of General Medicine, SCB Medical College and Hospital, Cuttack. ${ }^{2}$ Associate Professor, Department of General Medicine, SCB Medical College and Hospital, Cuttack.

\section{ABSTRACT}

\section{BACKGROUND}

The presence of cardiocirculatory dysfunction in liver cirrhosis has been described since 1960s, but it was erroneously attributed to alcoholic cardiomyopathy. Only in the last 2 decades, has it been shown that cardiac dysfunction is also present in nonalcoholic cirrhosis indicating the presence of a latent cardiac dysfunction known as "cirrhotic cardiomyopathy."

The aim of the study is to evaluate the cardiovascular dysfunction in nonalcoholic cirrhosis of liver and its correlation with the severity of cirrhosis of liver.

\section{MATERIALS AND METHODS}

In the descriptive study, 70 patients of nonalcoholic cirrhosis liver were taken up and were assessed for detection of cirrhosis and its complications and classified by Child-Pugh class. Cardiological function was evaluated in these patients by various parameters like clinical, ECG, Doppler echocardiography (systolic function, diastolic function, cardiac output and cardiac index) and proBNP and association with severity of cirrhosis of liver was assessed.

\section{RESULTS}

Cardiovascular evaluation of the patients in the study revealed ECG abnormality as QTc interval prolongation in 47 patients (67.1\%). Echocardiography revealed $51.4 \%$ patients having decreased ejection fraction and $40 \%$ patients had decreased fractional shortening. 29 patients (41.4\%) had stage I diastolic dysfunction (impaired relaxation) and 28 patients (40\%) were found to be in stage II diastolic dysfunction. Increase in serum proBNP levels was found in 20 patients (28.6\%), cardiac output and cardiac index were decreased in 18 patients (25.7\%) each. On analysing for correlation and associations, it was found that patients of cirrhosis having ECG abnormalities (QTc interval prolongation), increased systolic and diastolic dysfunction, increased left atrial diameter, decreased cardiac output, decreased cardiac index and increase in proBNP levels were significantly associated ( $p$ value $<0.05$ ) with increased Child-Pugh class, increased presence of hepatorenal syndrome, spontaneous bacterial peritonitis and hyponatraemia in cirrhotic patients.

\section{CONCLUSION}

This study showed the presence of cardiological dysfunction in nonalcoholic cirrhotic patients and a statistically significant association of the cardiological dysfunction with the severity of cirrhosis, which revealed the latent entity known as cirrhotic cardiomyopathy. Studies of cardiovascular abnormalities in cirrhosis of liver are scarce in literature, though other organ dysfunctions are well established. Hence, all cases of cirrhosis of liver should be periodically evaluated for presence of cardiac dysfunction, so that early intervention will reduce long-term morbidity and mortality.

\section{KEYWORDS}

Nonalcoholic Cirrhosis, Cirrhotic Cardiomyopathy, Doppler Echocardiography, QTc Interval Prolongation, Diastolic Dysfunction.

HOW TO CITE THIS ARTICLE: Bhuin S, Mohanty R. Evaluation and correlation of cardiovascular dysfunction in nonalcoholic cirrhotic patients with its severity. J. Evolution Med. Dent. Sci. 2017;6(73):5200-5204, DOI: 10.14260/Jemds/2017/1130

\section{BACKGROUND}

The presence of cardiocirculatory dysfunction in liver cirrhosis has been described since 1960s, but it was erroneously attributed to alcoholic cardiomyopathy, which is usually a dilated cardiomyopathy characterised by increased ventricular volumes and a decreased ejection fraction. Only in the last 2 decades has it been shown that cardiac dysfunction is also present in nonalcoholic cirrhosis.

Financial or Other, Competing Interest: None.

Submission 04-08-2017, Peer Review 27-08-2017,

Acceptance 02-09-2017, Published 11-09-2017.

Corresponding Author:

Dr. Subhas Bhuin

Flat J-2, Ashok Park Apartment, 268, N.S.C. Bose Road,

Bansdroni, Kolkata-700040, West Bengal.

E-mail: subhas8945@gmail.com

DOI: $10.14260 /$ jemds $/ 2017 / 1130$
Indicating the presence of a latent cardiac dysfunction known as "cirrhotic cardiomyopathy", which is defined at present as baseline increased cardiac output, but blunted ventricular response to stimuli, systolic and/or diastolic dysfunction, absence of overt left ventricular failure at rest, electrophysiological abnormalities including prolonged QT interval on electrocardiography and chronotropic incompetence.

It is a relatively new concept and has a negative prognostic impact, especially on the outcome of invasive procedures such as surgery, Transjugular Intrahepatic Portosystemic Shunt (TIPS) insertion and liver transplantation. Limited information is available about the epidemiology, prevalence and the natural history of the disease as its diagnosis is difficult because of near normal cardiac function at rest and majority are diagnosed during phases of clinical decompensation of 
cirrhosis in which they present with features of diastolic heart failure and/or high-output heart failure. ${ }^{1-4}$

The examination of cardiac function by echocardiography may initially be interpreted as normal cardiac function. On closer examination, both systolic and diastolic dysfunction (caused by decreased left ventricular compliance and relaxation) maybe demonstrated. Doppler echocardiography directed to detect simple echocardiographic indices, such as a reduced early/late filling velocity ratio ( $\mathrm{E} / \mathrm{A}$ ratio $\geq 1$ ), has been proposed as an available screening test for the diagnosis of the syndrome. Cirrhotic cardiomyopathy with refractory ascites is an important determinant and contribute to the pathogenesis of Hepatorenal Syndrome (HRS) precipitated by spontaneous bacterial peritonitis. Patients with suppressed cardiac function, decreased cardiac output and cardiac index had higher probability of developing HRS type 1 within 3 months supporting the association between cardiac dysfunction and renal failure in cirrhosis, the so-called "cardiorenal syndrome."5,6

The exact prognosis of cirrhotic cardiomyopathy remains unclear. The weight of evidence indicates that the clinical features of the syndrome become more prominent or worsen in parallel with progression of liver failure. E/A ratio $<1$, an indicator of diastolic dysfunction was predictive of slow ascites clearance and death after TIPS. These data point out the importance of a careful cardiac assessment in liver transplantation candidates, but also suggest the need for further studies to identify standardised diagnostic protocols and clear prognostic factors in this patient population. ${ }^{3}$ Cirrhotic cardiomyopathy being a relatively nascent topic with only few studies available in India and because of its mild subclinical course, this entity remains uncertain. Hence, this study of cardiovascular dysfunction in nonalcoholic cirrhosis is being undertaken to find the association between the cardiovascular abnormalities in nonalcoholic cirrhosis and its relation to the severity of the disease, so that early detection and treatment of cardiac abnormalities would help to favourably modify the course of cirrhosis of liver.

\section{Study}

The study was conducted on 70 indoor patients of nonalcoholic cirrhosis of liver admitted to PG Department of Medicine of SCB Medical College, Cuttack, over 1 year.

\section{Inclusion Criteria}

Patients diagnosed with cirrhosis of liver, between 18 and 80 years.

\section{Exclusion Criteria}

Alcoholic liver disease and patients with history of alcohol intake, history or presence of diabetes mellitus, systemic arterial hypertension, dyslipidaemia, coronary artery disease, structural, valvular and congenital heart disease, thyroid disorder, chronic kidney disease, severe or decompensated lung disease, presence of shock, history of liver disease that may also effect cardiac function (haemochromatosis, Wilson disease, amyloidosis), severe anaemia $(\mathrm{Hb}<7 \mathrm{~g} / \mathrm{dL})$ and pregnancy.

\section{Study Method}

In the descriptive study, 70 patients of nonalcoholic cirrhosis liver were taken. USG of liver was done for detection of cirrhosis and ascites with special reference to caudate lobe, portal vein and spleen. Ascites was graded clinically and by ultrasonography according to The International Ascites Club who has proposed the ascites grading system, Grade 1- mild ascites detectable only by ultrasound; Grade 2- moderate ascites with moderate symmetrical distention of abdomen; Grade 3- large or gross ascites with marked abdominal distention. EEG was done and grading of hepatic encephalopathy was done accordingly with West Haven Criteria for mental state examination. Evaluation for presence of Spontaneous Bacterial Peritonitis (SBP) and hepatorenal syndrome were done with clinical, blood and urine examination and ascitic fluid examination according to the diagnostic criteria. All patients were assessed regarding the severity of cirrhosis liver and classified by Child-Pugh Class. Cardiological function was evaluated in these patients by various parameters like clinical, ECG (corrected QT interval was calculated), echocardiography (systolic function, diastolic function, cardiac output and cardiac index) and proBNP. For the systolic and diastolic function of the left ventricle, following measurements were done (in four chamber view).

\section{Systolic Dysfunction}

End-diastolic volume, end-systolic volume, stroke volume, ejection fraction, Left Ventricular Internal Dimensions in systole (LVIDs), Left Ventricular Internal Dimensions in diastole (LVIDd), fraction shortening (FS) of internal diameter, Interventricular Septum Thickness (IVST), Left Ventricular Posterior wall diameter (LVP wed), cardiac output and cardiac index.

\section{Diastolic Dysfunction}

Mitral 'E' velocity (peak velocity of early mitral flow), mitral ' $A$ ' velocity (peak velocity of late mitral flow), E/A ratio (velocity of diastolic early filling wave/velocity of late filling wave), annular tissue motion (denoted by e'), E/e' ratio, Isovolumic Relaxation Time (IVRT), deceleration time of mitral ' $\mathrm{E}$ ' curve (DT of E) and left atrial diameter was calculated to find the presence of left atrial enlargement. The cardiac function abnormalities were compared and association with severity of cirrhosis of liver was assessed.

\section{Stages of Diastolic Dysfunction Stage 0 (Normal)}

E/A ratio between 1 and 2, e'>8-10, EDT between 150 and 190 $\mathrm{ms}$, IVRT between 60 and $90 \mathrm{~ms}$ and left atrial diameter $(<4.1$ $\mathrm{cm}$ in men and $<3.9 \mathrm{~cm}$ in women).

Stage 1 (Impaired Relaxation)

E/A ratio $<1, \mathrm{e}^{\prime}<8-10, \mathrm{E} / \mathrm{e}^{\prime}<8$.

\section{Stage 2 (Pseudonormal)}

E/A ratio between 1 and 1.5, e'<8-10, E/e' = 9-12, IVRT $>90$ $\mathrm{ms}$, mild-to-moderate left atrial enlargement $(4.1-5.1 \mathrm{~cm}$ in men and 3.9-4.6 cm in women). 


\section{Stages 3-4 (Restrictive)}

E/A ratio $>1.5, e^{\prime}<8-10, E / e^{\prime}>13$ and at least two of the following- (a) EDT $<150 \mathrm{~ms}$ (b) IVRT $<70$ (c) severe left atrial enlargement ( $\geq 5.2 \mathrm{~cm}$ in men and $\geq 4.7 \mathrm{~cm}$ in women).

Written informed consent was taken from patients and studied protocol was approved by institutional ethics committee for human research, SCB Medical College, Cuttack.

\section{Statistical Analysis}

Analysis was done using SPSS Version 21. Results on continuous measurements are presented on mean \pm SD (Min.Max.) and compared across groups using One-Way ANOVA test and independent T-test. Chi-square test has been used to find the significance of study parameters on categorical scale between two or more groups. P value of $<0.05$ was considered statistically significant.

\section{RESULTS}

The mean age of the patients in our study was $45 \pm 7.5$ years with 25 patients (35.7\%) in 30-40 years age group, 26 patients $(37.1 \%)$ in $41-50$ years age group and 19 patients $(27.1 \%)$ in 51 to 60 years age group. Patients were predominantly female; 38 female patients $(54.29 \%)$ to 32 male patients $(45.71 \%)$. Severity of the disease was assessed by Child-Pugh class, which revealed that out of 70 patients of nonalcoholic cirrhosis, 4 patients (5.7\%) were in Child-Pugh class A, 38 patients $(54.3 \%)$ were Child-Pugh class B and 28 patients (40\%) belonged to Child-Pugh class. In our study population, 15 patients $(21.4 \%)$ had mild ascites, 38 patients (54.3\%) had moderate ascites and 17 patients (24.3\%) had massive ascites according to The International Ascites Club Criteria. Hepatic encephalopathy was present in 57 patients (81.4\%) and absent in rest $13(18.6 \%)$ patients among which 14 patients (20\%) were found to have grade I, 22 patients (31.4\%) grade II, 17 (24.3\%) patients grade III and 4 patients (5.7\%) had grade IV hepatic encephalopathy. 24 patients (34.3\%) of the present study were found to have hepatorenal syndrome according to the diagnostic criteria of hepatorenal syndrome, whereas 10 patients $(14.3 \%)$ out of 70 patients were diagnosed to have spontaneous bacterial peritonitis. Cardiovascular evaluation of the patients in the study revealed ECG abnormality as QTc interval prolongation in 47 patients (67.1\%) out of 70 patients. 36 patients $(51.4 \%)$ were found to have decreased ejection fraction and 28 patients (40\%) had decreased fractional shortening. Out of 70 patients, 13 patients (18.6\%) were found to have normal diastolic function (stage 0), whereas 29 patients (41.4\%) had stage I diastolic dysfunction (impaired relaxation) and 28 patients (40\%) had stage II diastolic dysfunction. Left atrial diameter was found to be $<34 \mathrm{~mm}$ in 10 patients (14.3\%), 34-39 mm in 32 patients $(45.7 \%)$ and $>39 \mathrm{~mm}$ in 28 patients $(40 \%)$. Diastolic dysfunction was found to be associated with severity of cirrhosis significantly ( $p$ value $<0.05$ ). The parameters of diastolic function showed significant association ( $p$ value $<0.05$ ) with severity of cirrhosis like A wave, DT (deceleration time), IVRT (isovolumic relaxation time) and left atrial diameter showed increase in value above normal range, whereas E/A ratio (velocity of diastolic early filling wave/velocity of late filling wave) and e' wave (annular tissue motion) were decreased below normal range with increase in
Child-Pugh class. E/e' ratio and E wave were not found to be statistically significantly $(p>0.05)$ correlated with Child-Pugh class severity. Cardiac output was decreased in 18 patients (25.7\%), normal in 39 patients (55.7\%) and increased in 13 patients $(18.6 \%)$, whereas in 70 patients, 18 patients $(25.7 \%)$ had decreased cardiac index, 26 patients (37.1\%) had normal cardiac index and 26 patients $(37.1 \%)$ had increased cardiac index. ProBNP level in patients of cirrhosis was studied, which revealed increase in serum proBNP levels in 20 patients (28.6\%) out of 70 patients of the study. On analysing for correlation and associations, it was found that patients of cirrhosis having ECG abnormalities (QTc interval prolongation), increased systolic dysfunction, increased diastolic dysfunction, decreased cardiac output, decreased cardiac index and increase in proBNP levels were significantly associated ( $\mathrm{p}$ value $<0.05$ ) with increased Child-Pugh class, increased severity of ascites, increase grade of hepatic encephalopathy, presence of hepatorenal syndrome, presence of spontaneous bacterial peritonitis, hyponatraemia and hence increased severity of cirrhosis.

\begin{tabular}{|c|c|c|c|}
\hline \multicolumn{4}{|c|}{$\begin{array}{l}\text { Distribution of the Patients Based on } \\
\text { Systolic Function Parameters }(\mathrm{N}=70)\end{array}$} \\
\hline \multicolumn{2}{|c|}{ Systolic Parameters } & Frequency & Percent \\
\hline \multirow{2}{*}{ EDV } & Abnormal & 64 & 91.4 \\
\hline & Normal & 6 & 8.6 \\
\hline \multirow{2}{*}{ ESV } & Abnormal & 65 & 92.9 \\
\hline & Normal & 5 & 7.1 \\
\hline \multirow{3}{*}{$\mathrm{EF}$} & Increased & 4 & 5.7 \\
\hline & Normal & 30 & 42.9 \\
\hline & Decreased & 36 & 51.4 \\
\hline \multirow{3}{*}{ LVIDd } & Increased & 28 & 40 \\
\hline & Normal & 41 & 58.6 \\
\hline & Decreased & 1 & 1.4 \\
\hline \multirow{3}{*}{ LVIDs } & Increased & 28 & 40 \\
\hline & Normal & 40 & 57.1 \\
\hline & Decreased & 2 & 2.9 \\
\hline \multirow{3}{*}{ FS } & Increased & 5 & 7.1 \\
\hline & Normal & 37 & 52.9 \\
\hline & Decreased & 28 & 40 \\
\hline \multirow{2}{*}{ IV Sed } & Increased & 59 & 84.3 \\
\hline & Normal & 11 & 15.7 \\
\hline \multirow{2}{*}{ LVP Wed } & Increased & 57 & 81.4 \\
\hline & Normal & 13 & 18.6 \\
\hline \multicolumn{4}{|c|}{$\begin{array}{l}\text { Table 1. Echocardiographic Evaluation } \\
\text { of Systolic Function Parameters in the } \\
\text { Present Study Population of Liver Cirrhosis }\end{array}$} \\
\hline
\end{tabular}

\begin{tabular}{|c|c|c|c|c|}
\hline \multicolumn{5}{|c|}{ Child-Pugh Class Versus Diastolic Dysfunction } \\
\hline \multirow{2}{*}{$\begin{array}{c}\text { Child-Pugh } \\
\text { Class }\end{array}$} & \multicolumn{3}{|c|}{ Diastolic Dysfunction Grade } & \multirow{2}{*}{ P value* } \\
\hline & Grade 0 & Grade I & Grade II & \\
\hline A & $3(23.08)$ & $1(3.45)$ & $0(0)$ & \multirow{4}{*}{0.0204} \\
\hline B & $8(61.54)$ & $16(55.17)$ & $14(50)$ & \\
\hline $\mathrm{C}$ & $2(15.38)$ & $12(41.38)$ & $14(50)$ & \\
\hline Total & $13(100)$ & $29(100)$ & $28(100)$ & \\
\hline & Corr & on of Dia & Dysfur & \\
\hline
\end{tabular}

Figures in the parenthesis are percentages. *Chi-square test. 


\begin{tabular}{|c|c|c|c|c|c|}
\hline \multicolumn{6}{|c|}{ Comparison of Diastolic Cardiac Function Among Different Classes of Child-Pugh Score } \\
\hline \multirow{2}{*}{ Diastolic Cardiac Function Parameters } & & Child-Pugh Class & & \\
\hline & A (N=4) & B (N=38) & C (N=28) & F statistic & P value* \\
\hline & Mean \pm SD & Mean \pm SD & Mean \pm SD & & \\
\hline E wave & $80.5 \pm 15.0$ & $71.03 \pm 10.98$ & $71.07 \pm 9.87$ & 1.455 & 0.241 \\
\hline A wave & $72.5 \pm 1.0$ & $84.03 \pm 5.95$ & $89.43 \pm 6.64$ & 15.936 & $<0.001$ \\
\hline E/A & $1.11 \pm 0.22$ & $0.85 \pm 0.17$ & $0.79 \pm 0.13$ & 6.691 & 0.002 \\
\hline $\mathrm{e}^{\prime}$ & $11.30 \pm 5.62$ & $8.98 \pm 2.86$ & $8.08 \pm 2.23$ & 2.566 & 0.084 \\
\hline E/e' & $8.75 \pm 5.32$ & $8.25 \pm 1.73$ & $9.15 \pm 1.99$ & 1.451 & 0.241 \\
\hline DT (ms) & $176.0 \pm 22.69$ & $201.58 \pm 13.96$ & $212.89 \pm 15.84$ & 11.964 & $<0.001$ \\
\hline IVRT (ms) & $76.0 \pm 14.70$ & $101.0 \pm 14.63$ & $111.29 \pm 7.72$ & 16.320 & $<0.001$ \\
\hline LA diameter & $33.50 \pm 1.91$ & $37.08 \pm 3.55$ & $41.32 \pm 4.23$ & 13.877 & $<0.001$ \\
\hline Table 3. Comparison of Diastolic Cardiac Function Among Different Classes of Child-Pugh Score
\end{tabular}

*ANNOVA test.

\begin{tabular}{|c|c|c|c|c|}
\hline \multicolumn{5}{|c|}{ Comparison of Liver Function Tests Versus ECG Changes } \\
\hline \multirow{3}{*}{ LFT Parameters } & \multicolumn{4}{|c|}{ ECG Changes } \\
\hline & Normal (n=23) & QTc Inc. $(n=47)$ & T Statistic & P* Value \\
\hline & Mean \pm SD & Mean \pm SD & & \\
\hline S. bilirubin (total) & $2.37 \pm 0.31$ & $3.0 \pm 0.71$ & -5.14 & $<0.001$ \\
\hline S. bilirubin (direct) & $1.17 \pm 0.13$ & $1.36 \pm 0.30$ & -3.926 & $<0.001$ \\
\hline AST & $200.91 \pm 159.5$ & $234.45 \pm 172.9$ & -0.803 & 0.426 \\
\hline ALT & $140.74 \pm 118.9$ & $166.55 \pm 122.8$ & -0.844 & 0.403 \\
\hline ALP & $382.26 \pm 77.2$ & $405.83 \pm 76.5$ & -1.204 & 0.235 \\
\hline S. protein & $5.70 \pm 0.19$ & $5.56 \pm 0.26$ & 2.532 & 0.014 \\
\hline S. albumin & $3.08 \pm 0.26$ & $2.54 \pm 0.46$ & 6.26 & $<0.001$ \\
\hline PT (test) secs. & $14.54 \pm 0.93$ & $18.18 \pm 2.92$ & -7.803 & $<0.001$ \\
\hline INR & $1.53 \pm 0.13$ & $2.14 \pm 0.49$ & -7.9 & $<0.001$ \\
\hline
\end{tabular}

*Independent T-test.

\begin{tabular}{|c|c|c|c|}
\hline \multicolumn{4}{|c|}{ Child-Pugh Class Versus ProBNP } \\
\cline { 1 - 3 } Child-Pugh Class & Inc. & N & \\
\cline { 2 - 3 } & $0(0.0)$ & 4 & \\
\hline A & $6(30.0)$ & 32 & \multirow{2}{*}{$0.004^{*}$} \\
\hline B & $14(70.0)$ & 14 & \\
\hline C & $\mathbf{2 0}(100.0)$ & $\mathbf{5 0}(100.0)$ & \\
\hline Total & \multicolumn{3}{|c|}{ Table 5. Correlation of ProBNP with Child-Pugh } \\
Class in Cirrhotic Patients of Present Study \\
\hline
\end{tabular}

Figures in the parenthesis are percentages.

${ }^{*}$ Chi-square test.

\begin{tabular}{|c|c|c|c|c|}
\hline \multicolumn{3}{|c|}{ Spontaneous Bacterial Peritonitis Versus Cardiac Index } \\
\hline \multirow{2}{*}{ SBP } & \multicolumn{3}{|c|}{ Cardiac Index } & N value \\
\cline { 2 - 4 } & Decreased & Normal & Increased & \\
\hline Absent & $8(44.4)$ & $26(100.0)$ & $26(100.0)$ & \multirow{2}{*}{$0.001^{*}$} \\
\hline Present & $10(55.6)$ & $0(0.0)$ & $0(0.0)$ & \\
\hline Total & $\mathbf{1 8}(100.0)$ & $\mathbf{2 6}(100.0)$ & $\mathbf{2 6}(100.0)$ & \\
\hline \multicolumn{3}{|c|}{ Table 6. Correlation of Cardiac Index with SBP } \\
in Cirrhotic Patients of Present Study \\
\hline
\end{tabular}

Figures in the parenthesis are percentages.

${ }^{*}$ Chi-square test.

\begin{tabular}{|c|c|c|c|c|}
\hline \multicolumn{5}{|c|}{ Hepatorenal Syndrome Versus Cardiac Index } \\
\hline \multirow{2}{*}{\begin{tabular}{|c} 
Hepatorenal \\
Syndrome
\end{tabular}} & \multicolumn{3}{|c|}{ Cardiac Index } & \multirow{2}{*}{$\begin{array}{c}\mathbf{P} \\
\text { Value }\end{array}$} \\
\hline & Decreased & Normal & Inc & \\
\hline Absent & & 20 & 260 & \multirow{3}{*}{$0.001^{*}$} \\
\hline \multirow[t]{2}{*}{ Present } & $18(100.0)$ & $6(2$ & $0(0.0)$ & \\
\hline & $18(100.0)$ & $26(100.0)$ & $26(100.0)$ & \\
\hline & & & S & \\
\hline
\end{tabular}

Figures in the parenthesis are percentages.

*Chi-square test.

\section{DISCUSSION}

In the present study, cardiac dysfunction in nonalcoholic cirrhosis has been studied in detail, which shows QTc interval prolongation in 47 patients (67.1\%), systolic dysfunction in 37 patients (52.9\%), diastolic dysfunction in 57 patients (81.4\%), decreased cardiac output and decreased cardiac index in 18 patients $(25.7 \%)$ and increased serum proBNP in 20 patients $(28.6 \%)$ out of 70 patients of the study, which is statistically significantly associated with severity of cirrhosis. Study by Ruiz-del-Arbol et al reported a lower cardiac output in patients with cirrhosis who developed renal failure during a course of spontaneous bacterial peritonitis compared to those without renal failure. Studies by Valeriano et al, Mohammad Nasr et al and Inucci et al found significant increases in A wave velocity, DT and IVRT with decreases in E/A ratio in the Child-Pugh class B and C cirrhotic patients. Moller and Henriksen and Finucci et al reported that cirrhotic patients have increased left ventricular end-diastolic diameters. Pozzi et al found that in cirrhotic patients with tense ascites, the A wave velocity is markedly increased, the $\mathrm{E} / \mathrm{A}$ ratio is markedly reduced and the deceleration time is significantly prolonged, whereas Wong et al reported prolonged isovolumic relaxation time in cirrhotic patients irrespective of the presence of ascites with significantly reduced E/A ratios only in ascitic subjects. E/A ratio $<1$, an indicator of diastolic dysfunction was found to be predictive of slow ascites clearance and death after TIPS. Study by Sunil Dadhich et al showed left ventricular diastolic dysfunction (type I and II) was seen in $80 \%$ of cirrhotic patients with ascites as compared to $60 \%$ of cirrhotic patients without ascites. ${ }^{6-9}$ Studies by JH Henriksen et al and Vi Nguyen et al showed the circulating proBNP concentration is 
significantly increased in patients with advanced cirrhosis and a close relation was found between cirrhosis with increased ascites with BNP level. They postulated that raised BNP and proBNP levels could serve to identify early cardiac dysfunction in patients with cirrhosis. ${ }^{10}$ Hence, this present study was found to be in accordance to other above-mentioned studies on cardiovascular dysfunction in cirrhosis.

\section{CONCLUSION}

Studies of cardiovascular abnormalities in cirrhosis of liver are scarce in literature though other organ dysfunctions are well established. The few studies that have been done in these patients have led to the new terminology of "cirrhotic cardiomyopathy", which indicates a chronic ongoing process leading to cardiac dysfunction in cirrhosis of liver. Studies on cardiac dysfunction in nonalcoholic cirrhosis is still rarer. Therefore, all cases of cirrhosis of liver should be periodically evaluated for presence of cardiac dysfunction, so that early intervention will reduce long-term morbidity and mortality.

\section{REFERENCES}

[1] Bacon BR. Cirrhosis and its complications. Liver and biliary tract disease. Harrison's principles of internal medicine. 19th edn. McGraw-Hill 2015;2(14):2058-66.

[2] Fede G, Privitera G, Tomaselli T, et al. Cardiovascular dysfunction in patients with liver cirrhosis. Annals of Gastroenterology 2014;27:1-10.

[3] Cazzaniga MF, Salerno G, Pagnozzi G, et al. Diastolic dysfunction is associated with poor survival in patients with cirrhosis with transjugular intrahepatic portosystemic shunt. Gut 2007;56(6):869-75.
[4] Zardi EM, Abbate A, Zardi DM, et al. Cirrhotic cardiomyopathy. J Am Coll Cardiol 2010;56(7):539-49.

[5] Ruiz-del-Arbol L, Monescillo A, Arocena C, et al. Circulatory function and hepatorenal syndrome in cirrhosis. Hepatology 2005;42(2):439-47.

[6] Pozzi M, Carugo S, Boari G, et al. Evidence of functional and structural cardiac abnormalities in cirrhotic patients with and without ascites. Hepatology 1997;26(5):1131-7.

[7] Wong FN, Girgrah N, Graba J, et al. The cardiac response to exercise in cirrhosis. Gut 2001;49(2):268-75.

[8] Nasr FM, Metwaly A, Khalik AA, et al. Cardiac dysfunction in liver cirrhosis: a tissue Doppler imaging study from Egypt. Electron Physician 2015;7(4): 1135-43.

[9] Papastergiou V, Skorda L, Lisgos P, et al. Ultrasonographic prevalence and factors predicting left ventricular diastolic dysfunction in patients with liver cirrhosis: is there a correlation between grade of diastolic dysfunction and grade of liver disease? Scientific World Journal Article ID 615057, 2012; 2012: p. 6.

[10] Vi Nguyen, Zielinski R, Harnett P, et al. NT-proBNP Changes in patients with ascites during large volume paracentesis. ISRN Hepatology Article ID 959474, 2013;2013: p. 7. 